\title{
Ethique clinique: L'importance d'être non-autoritaire
}

\section{Jean Martin}

Membre de la rédaction, ancien membre de la Commission nationale d'éthique

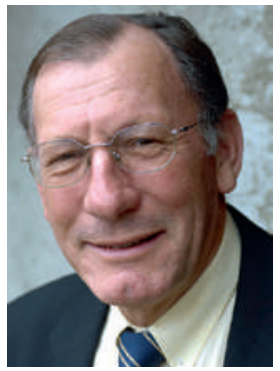

«Dans le dialogue national sur la formation et la certification des consultants en éthique clinique (CEC), la demande est universelle pour améliorer leurs connaissances et compétences. Il est largement admis que beaucoup d'entre eux ne sont pas adéquatement formés.» C'est le début d'un article du Hastings Center Report, par la directrice du Programme de médiation en éthique clinique de l'Université de Pennsylvanie [1]. Qui poursuit: «Il y a un consensus clair qu'une attitude 〈autoritaire` doit être évitée dans le conseil éthique -à savoir, éviter une approche où le professionnel impose ses priorités éthiques ou ses convictions religieuses à l'ensemble des «stakeholders` (personnes concernées) dans une situation complexe ou conflictuelle.»

Pourtant, dit Fiester, bien peu a été fait jusqu'ici pour former ces professionnels à ne pas imposer leurs valeurs. "Une étape essentielle est d'identifier ce qui est ses convictions personnelles, voire ses souhaits normatifs, dans le contexte de la diversité des positions, en soi justifiables mais contrastées, qu'on trouve dans le pluralisme propre aux Etats-Unis. Evaluer sa propre position parmi d'autres points de vue légitimes fait prendre du recul par rapport à des engagements moraux qu'on peut personnellement juger absolus ou universels» (alors qu'ils ne le sont pas pour d'autres). Il paraît clair que, dans les pays européens dont les fondements philosophiques et juridiques sont comparables, le même avertissement est valable.

L'article de Fiester décrit un «Bioethical Positions Inventory", comprenant quinze paires d'affirmations sur des questions éthiques (dans chaque paire, une attitude "conservatrice» et une «libérale»). Les professionnels en formation font d'abord un diagnostic de leur propre position sur chaque question puis, dans un second temps, leur tâche est de préparer une argumentation défendant la position à laquelle ils sont opposés! (Cet exercice les protège de s'en remettre à une discutable «hégémonie des valeurs» - Fiester.)

Fiester A. Teaching Nonauthoritarian Clinical Ethics: Using an Inventory of Bioethical Positions. Hastings Center Report. 2015; 45(2):20-6.

jean.martin[at]saez.ch
On leur demande aussi de discuter une expérience vécue, selon les étapes suivantes: 1) Décrivez une consultation où une position à laquelle vous êtes profondément attaché était en opposition directe avec celle du patient, cas échéant de la famille ou d'autres soignants; comment alors avez-vous conduit la discussion?; 2) Décrivez un cas où vous avez été préoccupé de réaliser que vous orientiez la consultation pour aligner son résultat avec vos valeurs à vous? Avez-vous fait quelque chose pour corriger cela?; 3) Décrivez un cas où une des personnes concernées a eu de la peine à s'exprimer et donner sa position morale. Quelle a été votre stratégie pour "amplifier» et articuler son opinion devant le groupe? Ce faisant, comment avez-vous maintenu votre propre neutralité quant aux valeurs en cause? De telles démarches pédagogiques sont pertinentes et nécessaires. En 2012, l'Académie Suisse des Sciences Médicales (www.samw.ch), dans sa recommandation "Soutien éthique en médecine» et à propos de l'attitude fondamentale des membres d'une structure d'éthique, dit entre autres: «faire preuve d'introspection sur leurs propres choix de valeur; être prêts à reconsidérer leur avis initial à la lumière d'une discussion commune, [notamment] avec des personnes de points de vue différents; être ouverts aux autres disciplines et professions; être prêts à acquérir une vision réaliste du quotidien clinique.»

Cela étant, la mise en œuvre d'une pratique de sélection et de formation de membres de commissions d'éthique en accord avec ce qui précède n'est certainement pas simple (sans même parler ici d'évaluation/supervision ultérieure). Fiester encore: "Les organisations nationales de bioéthique ont émis des avertissements à propos du risque d'imposition de leurs valeurs par les consultants en éthique, mais le problème est trop insidieux pour être amélioré par de simples directives.» Oui, on est ici dans un domaine qui ne se laisse guère gérer par des règlements et est plus du registre des attitudes professionnelles, relationnelles, déontologiques. C'est plus par la réflexion et l'échange interdisciplinaire approfondi sur des situations difficiles qu'on peut promouvoir une culture non-autoritaire en éthique. L'auteure parle de "capacité de bien écouter et de montrer à l'autre (aux autres) intérêt, respect, soutien et empathie». Il convient d'avoir ces enjeux à l'esprit, dans le travail au sein de commissions d'éthique comme dans la pratique quotidienne.

N. B:

Cet article parle de la situation aux Etats-Unis, où souvent les CEC ne sont pas médecins. Toutefois, l'argumentation développée vaut pour toutes celles et ceux qui sont engagés dans la consultation éthique clinique (on reconnaît dans nos pays aussi que le conseil éthique ne peut/doit pas être un «monopole» médical). 